\title{
Effects of short-term treatment with atorvastatin in smokers with asthma - a randomized controlled trial
}

\author{
Georgina Braganza', Rekha Chaudhuri ${ }^{1}$, Charles McSharry², Christopher J Weir ${ }^{3}$, Iona Donnelly², Lisa Jolly ${ }^{2}$, \\ Jane Lafferty ${ }^{1}$, Suzanne M Lloyd ${ }^{4}$, Mark Spears ${ }^{1}$, Frances Mair ${ }^{5}$ and Neil C Thomson ${ }^{{ }^{*}}$
}

\begin{abstract}
Background: The immune modulating properties of statins may benefit smokers with asthma. We tested the hypothesis that short-term treatment with atorvastatin improves lung function or indices of asthma control in smokers with asthma.

Methods: Seventy one smokers with mild to moderate asthma were recruited to a randomized double-blind parallel group trial comparing treatment with atorvastatin ( $40 \mathrm{mg}$ per day) versus placebo for 4 weeks. After 4 weeks treatment inhaled beclometasone ( $400 \mu \mathrm{g}$ per day) was added to both treatment arms for a further 4 weeks. The primary outcome was morning peak expiratory flow after 4 weeks treatment. Secondary outcome measures included indices of asthma control and airway inflammation.
\end{abstract}

Results: At 4 weeks, there was no improvement in the atorvastatin group compared to the placebo group in morning peak expiratory flow [-10.67 L/min, $95 \% \mathrm{Cl}-38.70$ to $17.37, \mathrm{p}=0.449]$, but there was an improvement with atorvastatin in asthma quality of life score [0.52, 95\% Cl 0.17 to $0.87 \mathrm{p}=0.005]$. There was no significant improvement with atorvastatin and inhaled beclometasone compared to inhaled beclometasone alone in outcome measures at 8 weeks.

Conclusions: Short-term treatment with atorvastatin does not alter lung function but may improve asthma quality of life in smokers with mild to moderate asthma.

Trial Registration: Clinicaltrials.gov identifier: NCT00463827

\section{Background}

Smokers with asthma have poor symptom control, accelerated decline in lung function and an attenuated response to inhaled corticosteroids compared to nonsmokers with asthma[1-4]. Optimum medical therapy for smokers with asthma has not been established. There is an unmet need for alternative or additional drugs for smokers with asthma who are unable to stop smoking.

In addition to reducing cholesterol biosynthesis by inhibiting 3-hydroxy-3-methylgluteryl coenzyme A (HMG-CoA) reductase, statins have pleiotropic anti-

\footnotetext{
* Correspondence: neil.thomson@glasgow.ac.uk

${ }^{1}$ Respiratory Medicine, Institute of Infection, Immunity \& Inflammation,

University of Glasgow, Glasgow, UK

Full list of author information is available at the end of the article
}

inflammatory effects[5] that may be beneficial in the treatment of chronic inflammatory diseases[5,6]. Preclinical in vitro and in vivo studies, including experimental models of allergic lung inflammation[7,8], have shown that statins reduce components of airway inflammation potentially relevant to the pathogenesis of asthma. Despite preliminary data showing an absence of clinical benefit with short-term statin therapy on asthma symptoms or lung function in non-smokers with asthma [9-11], there is indirect evidence to suggest that statins may show efficacy in patients with asthma who smoke. First, simvastatin inhibits lung parenchymal destruction, peribronchial and perivascular inflammatory cell infiltrate and leads to a reduction in levels of matrix metalloproteinase- 9 in a rat model of smoking related lung disease[12] and reduces airway inflammation and

\section{Ciomed Central}


reverses elastase-induced pulmonary emphysema in mice[13]. Second, statins enhance corticosteroid sensitivity in asthma[14,15], possibly by restoring histone deacetylase (HDAC) activity[16], which is reduced by cigarette smoking[17]. Thirdly, retrospective or case cohort studies have shown statin use is associated with a reduced decline in lung function in cigarette smokers [18] and reduced exacerbation rates, hospital admission and mortality in patients with COPD[19]. Taken together, this evidence suggests that statin treatment may improve asthma control in smokers with asthma. This study aimed to test the hypothesis that short term treatment with atorvastatin, either alone or in addition to low dose inhaled corticosteroid improves lung function or other indices of asthma control and/or airway inflammation in smokers with asthma. Atorvastatin was chosen because of its favourable in vitro and in vivo anti-inflammatory properties [20-22] and evidence of clinical benefit at the dose of $40 \mathrm{mg}$ daily in rheumatoid arthritis [21].

\section{Methods}

\section{Study subjects}

Cigarette smokers with chronic asthma symptoms of more than one year duration, aged 18 to 60 years were eligible to take part. Participants were regular smokers with a history of 5 pack years or more. Patients were recruited from general practice, hospital clinics and research databases. Smoking cessation advice was offered at the study screening visit and eligible subjects who were not willing to quit were enrolled. Patients were excluded if they were already taking a statin or medications known to interact with statins such as antifungal agents, macrolide antibiotics, cyclosporin, gemfibrozil, verapamil and amiodarone. All subjects demonstrated reversibility in $\mathrm{FEV}_{1}$ following salbutamol of $\geq 12 \%$ and $\geq 200 \mathrm{ml}$ or $\mathrm{PC}_{20} \leq 8 \mathrm{mg} / \mathrm{ml}$ or $\geq 20 \%$ variability in peak expiratory flow (PEF)[23]. The West Glasgow Research Ethics Committee approved the study and all patients gave written informed consent.

\section{Study design}

The aim of the study was to test the hypothesis that shortterm treatment with atorvastatin improves lung function or indices of asthma control or airway inflammation in smokers with asthma. Patients had been free of exacerbation and lower respiratory tract infection for a minimum period of 6 weeks prior to randomization. Where relevant, inhaled corticosteroid (ICS) and long-acting bronchodilator (LABA) treatment were weaned over a 4-10 week period. On completion of a two-week run in period free from ICS and LABA, a baseline visit was performed. Subjects recorded their PEF and asthma symptoms twice daily throughout the study. Randomization was conducted via an interactive telephone service (IVRS) in randomized blocks of length four. Patients were assigned to eight weeks of atorvastatin $40 \mathrm{mg}$ daily or a matched placebo. After four weeks inhaled beclometasone $\left(\mathrm{Clenil}^{\circledR}{ }^{\mathbb{2}} \mathrm{Modu}-\right.$ lite ${ }^{\circledR} 200 \mu \mathrm{g}$ twice daily) was also commenced in both randomized groups. Treatment with short-acting $\beta-2$ agonist was allowed throughout the study. At baseline, 4 and 8 week visits, electronic PEF data were downloaded and spirometry performed. At these visits patients completed a validated asthma control questionnaire (ACQ) and asthma quality of life questionnaire (AQLQ); induced sputum was performed and exhaled nitric oxide was measured. At baseline and 8 weeks, blood samples were taken for immunological tests, lipids and liver transaminases.

\section{Measurements}

PEF was recorded using Piko-1 electronic peak flow meters (nSpire Hertford UK) and symptoms were recorded in a validated diary card[24]. ACQ[25] and AQLQ [total and individual domains][26] results were also recorded. The total AQLQ score is the mean of all 32 responses and the individual domain scores are the means of the items in those domains. A change in score of 0.5 is the smallest change that is considered clinically important. Exhaled nitric oxide (NO) levels were measured at $50 \mathrm{ml} / \mathrm{s}$ in concordance with standardised guidelines[27] (Niox Flex, Aerocrine, Sweden). Spirometry was performed to American Thoracic Society guidelines. Exhaled carbon monoxide levels were measured by hand held Smokerlyser. (Bedford scientific, UK). Airway hyper-responsiveness was measured by Cockcroft's methacholine challenge test with concentrations from $0.03 \mathrm{mg} / \mathrm{ml}$ to $16 \mathrm{mg} / \mathrm{ml}[28]$. Blood samples were processed by local accredited laboratories for urea and electrolytes, liver transaminases, thyroid function, creatinine kinase levels and lipid profile; haematological testing (full blood count), and total IgE and specific IgE to cat, house dust mite and grass. Total IgE levels $>120 \mathrm{IU} / \mathrm{ml}$ was considered elevated and specific IgE levels $>0.35$ $\mathrm{AU} / \mathrm{ml}$ was used to define atopic status. Sputum induction was performed as previously described[29], using a low concentration of DTT $(0.003 \%)$ to disperse cells without undue effects on mediator measurements. Sputum supernatant fluid was analysed for leukotriene (LT) $\mathrm{B}_{4}$ and myeloperoxidase (MPO) using enzyme immunoassay (EIA) ( $\mathrm{LTB}_{4}$ from R\&D Systems, Abingdon, UK, MPO from Cambridge Bioscience, Cambridge, UK.), and interleukin (IL)-2, 4, 6, 8, 10 and tumor necrosis factor (TNF)- $\alpha$ using a Luminex microbead fluorescence kit (Biosource, Invitrogen, Paisley, UK). Serum was analysed for high sensitivity (hs)-CRP by EIA (R \& D Systems) and sICAM-1, E-selectin, P-selectin, IL-2, 4, 6, 8, 10 and TNF- $\alpha$ using a multiplex fluorescence bead kit (sICAM1, E-selectin and P-selectin from Merck Chemicals, 
Hertfordshire, UK. Interleukins and TNF- $\alpha$ by Biosource). Current smoking was confirmed by serum cotinine measurement (Cozart, Oxfordshire, UK). Treatment compliance was assessed by tablet count and inhaler weight. Adverse event information was reviewed at each study visit.

\section{Analysis}

Baseline characteristics were described by number and percentage of patients for categorical variables and mean (SD) or median and inter-quartile range for normally distributed or skewed continuous variables respectively. The full analysis set was defined as all randomized subjects with at least one post-baseline assessment of PEF (primary endpoint). The primary endpoint was calculated as the mean of the morning PEF measurements from the Piko-1 electronic peak flow meter recordings during the seven days immediately preceding each study visit. If fewer than three days of data were recorded within that period, patient diary entries for morning PEF were substituted in place of the missing values. The mean was then calculated if there were at least three days of data available, otherwise the response was taken to be missing. Response to atorvastatin on lung function, diary data, induced sputum, mediator levels and exhaled nitric oxide versus placebo was assessed by analysis of covariance, where the response was change from baseline and the baseline value was the covariate. All data were analysed using SAS version 9 (SAS Institute, Cary, NC). A sample size of 68 was calculated to have $80 \%$ power to detect a mean difference of $25 \mathrm{~L} / \mathrm{min}$ in change from baseline to four weeks in morning PEF, the primary endpoint[30], assuming a standard deviation of changes of $36 \mathrm{~L} / \mathrm{min}$, using a two sample t-test with a $5 \%$ two-sided significance level. Recruitment of 74 patients was planned to ensure that 68 patients completed the study.

\section{Results}

\section{Recruitment and baseline characteristics}

Of the 286 subjects contacted, 131 volunteers were consented and 71 were randomized to either the atorvastatin treatment group $(\mathrm{n}=36)$ or the placebo group $(\mathrm{n}=35)$ (Figure 1). Baseline demographic and clinical characteristics of patients are listed in Tables 1 and 2. The two groups were well balanced with respect to these characteristics.

\section{Changes in clinical outcomes}

Changes in clinical outcomes after atorvastatin treatment are listed in Table 2 and illustrated in Figure 2. At 4 weeks, the change in mean morning PEF, as compared with baseline, did not differ substantially between the atorvastatin and placebo treatment periods [mean

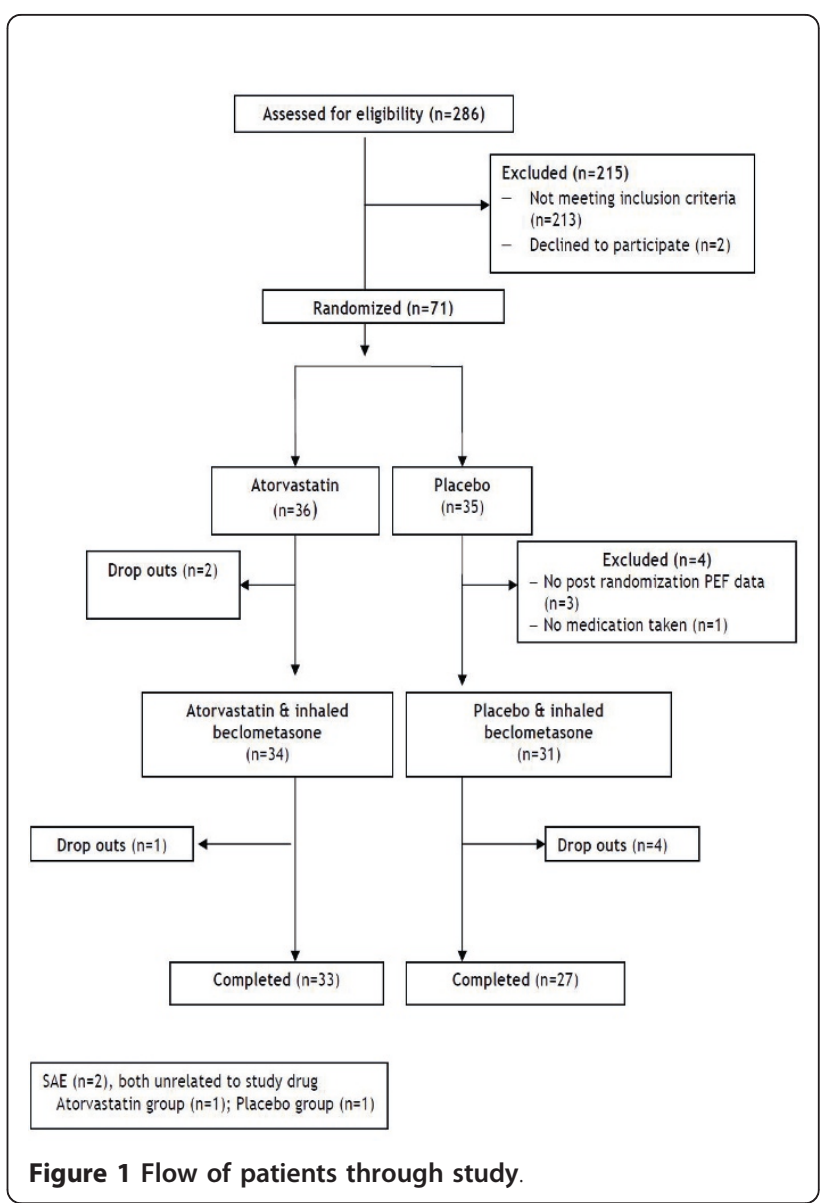

Table 1 Demographics and clinical baseline characteristics

\begin{tabular}{|c|c|c|}
\hline & $\begin{array}{l}\text { Atorvastatin } \\
(\mathrm{n}=36)^{\dagger}\end{array}$ & $\begin{array}{l}\text { Placebo } \\
(\mathrm{n}=32)^{\dagger}\end{array}$ \\
\hline Age, years & $\begin{array}{c}40.4 \\
(34.8,45.5)\end{array}$ & $\begin{array}{c}43.0 \\
(38.1,52.0)\end{array}$ \\
\hline Male sex, n (\%) & $15(41.7 \%)$ & $15(46.9 \%)$ \\
\hline Smoking history (pack years) & $24(14,34)$ & $21(10,30)$ \\
\hline Duration of asthma symptoms, years & $\begin{array}{c}24.0 \\
(14.5,32.5)\end{array}$ & $\begin{array}{l}19.5(12.5 \\
38.5)\end{array}$ \\
\hline Atopic, n (\%) & $18(54.5 \%)$ & $21(67.7 \%)$ \\
\hline Total lgE (IU/ml) & $82(19,192)$ & $197(76,517)$ \\
\hline $\begin{array}{l}\text { Use of inhaled corticosteroid at } \\
\text { screening, } n(\%)\end{array}$ & $19(52.8 \%)$ & $16(50.0 \%)$ \\
\hline $\begin{array}{l}\text { Equivalent beclometasone dose at } \\
\text { screening, } \mu \mathrm{g}\end{array}$ & $100(0,400)$ & $200(0,800)$ \\
\hline Use of LABA at screening, $n(\%)$ & $8(22.2 \%)$ & $14(43.8 \%)$ \\
\hline Pre-bronchodilator $\mathrm{FEV}_{1} \%$ predicted & $84(71,98)$ & $79(65,92)$ \\
\hline $\mathrm{FEV}_{1} \%$ reversibility & $11(6,16)$ & $12(8,19)$ \\
\hline Serum cotinine (ng/mL) & $85(78,90)$ & $82(79,94)$ \\
\hline
\end{tabular}

Definition of abbreviations: $\mathrm{FEV}_{1}$, Forced Expiratory Volume in one second; LABA, long-acting beta ${ }_{2}$-agonist.

Data represented as median (IQR) unless specified.

${ }^{\dagger}$ Number of randomized subjects with at least one post-baseline assessment of PEF. 
Table 2 Clinical outcomes after atorvastatin treatment or placebo

\begin{tabular}{|c|c|c|c|c|c|c|c|c|}
\hline \multirow[t]{2}{*}{ Variables } & \multicolumn{2}{|c|}{ Baseline } & \multicolumn{2}{|c|}{4 weeks } & \multirow{2}{*}{$\begin{array}{c}\text { †Treatment } \\
\text { difference, } \\
\text { atorvastatin } \\
\text { minus } \\
\text { placebo } \\
(95 \% \mathrm{Cl})\end{array}$} & \multicolumn{2}{|c|}{8 weeks } & \multirow{2}{*}{$\begin{array}{c}\text { †Treatment } \\
\text { difference, } \\
\text { atorvastatin } \\
\text { minus } \\
\text { placebo } \\
(95 \% \mathrm{Cl})\end{array}$} \\
\hline & $\begin{array}{l}\text { Atorvastatin } \\
(n=36)\end{array}$ & $\begin{array}{l}\text { Placebo } \\
(n=32)\end{array}$ & $\begin{array}{l}\text { Atorvastatin } \\
\quad(\mathrm{n}=34)\end{array}$ & $\begin{array}{l}\text { Placebo } \\
(n=30)\end{array}$ & & $\begin{array}{c}\text { Atorvastatin } \\
\& \text { ICS } \\
(n=33)\end{array}$ & $\begin{array}{c}\text { Placebo } \\
\& \text { ICS } \\
(n=27)\end{array}$ & \\
\hline Morning PEF L/min & $\begin{array}{l}381.33 \\
(138.79)\end{array}$ & $\begin{array}{c}348.99 \\
(116.49)\end{array}$ & $\begin{array}{c}376.39 \\
(141.63)\end{array}$ & $\begin{array}{c}357.65 \\
(121.38)\end{array}$ & $\begin{array}{c}-10.67 \\
(-38.70,17.37) \\
p=0.449\end{array}$ & $\begin{array}{c}390.51 \\
(123.38)\end{array}$ & $\begin{array}{c}354.64 \\
(123.70)\end{array}$ & $\begin{array}{c}10.68 \\
(-20.29,41.65) \\
p=0.492\end{array}$ \\
\hline Evening PEF L/min & $\begin{array}{c}391.9 \\
(137.1)\end{array}$ & $\begin{array}{c}375.8 \\
(118.4)\end{array}$ & $\begin{array}{c}384.8 \\
(137.1)\end{array}$ & $\begin{array}{c}367.4 \\
(117.2)\end{array}$ & $\begin{array}{c}4.95 \\
(-18.61,28.51) \\
p=0.676\end{array}$ & $\begin{array}{l}400.0 \\
(140.1)\end{array}$ & $\begin{array}{c}368.3 \\
(130.5)\end{array}$ & $\begin{array}{c}25.03 \\
(-2.04,52.11) \\
p=0.069\end{array}$ \\
\hline FEV $_{1}$ pre-salbutamol, L & $\begin{array}{l}2.76 \\
(0.91)\end{array}$ & $\begin{array}{l}2.51 \\
(0.76)\end{array}$ & $\begin{array}{l}2.79 \\
(0.83)\end{array}$ & $\begin{array}{l}2.59 \\
(0.74)\end{array}$ & $\begin{array}{c}-0.01 \\
(-0.13,0.11) \\
p=0.872\end{array}$ & $\begin{array}{l}2.80 \\
(0.89)\end{array}$ & $\begin{array}{l}2.63 \\
(0.76)\end{array}$ & $\begin{array}{c}0.03 \\
(-0.10,0.16) \\
p=0.651\end{array}$ \\
\hline $\mathrm{FEV}_{1}$ post-salbutamol, L & $\begin{array}{l}3.08 \\
(0.94)\end{array}$ & $\begin{array}{l}2.88 \\
(0.75)\end{array}$ & $\begin{array}{c}3.02 \\
(0.88)\end{array}$ & $\begin{array}{l}2.89 \\
(0.75)\end{array}$ & $\begin{array}{c}-0.03 \\
(-0.13,0.07) \\
p=0.492\end{array}$ & $\begin{array}{c}3.02 \\
(0.90)\end{array}$ & $\begin{array}{l}2.93 \\
(0.74)\end{array}$ & $\begin{array}{c}-0.02 \\
(-0.12,0.07) \\
p=0.642\end{array}$ \\
\hline $\begin{array}{c}\mathrm{PC}_{20} \text { methacholine } \\
\text { geometric mean, } \mathrm{mg} / \mathrm{ml}\end{array}$ & $\begin{array}{c}3.29 \\
(4.03)\end{array}$ & $\begin{array}{c}2.15 \\
(3.34)\end{array}$ & $\begin{array}{c}4.12 \\
(4.86)\end{array}$ & $\begin{array}{l}2.93 \\
(5.0)\end{array}$ & $\begin{array}{c}1.36 \\
(-1.29,4.01) \\
p=0.307\end{array}$ & $\begin{array}{l}5.31 \\
(6.2)\end{array}$ & $\begin{array}{l}2.31 \\
(4.0)\end{array}$ & $\begin{array}{c}1.37 \\
(-0.65,3.40) \\
p=0.179\end{array}$ \\
\hline $\begin{array}{c}\text { ACQ Score } \\
\text { [6 questions] }\end{array}$ & $\begin{array}{c}1.86 \\
(1.02)\end{array}$ & $\begin{array}{c}1.86 \\
(0.82)\end{array}$ & $\begin{array}{c}1.42 \\
(0.68)\end{array}$ & $\begin{array}{c}1.91 \\
(1.10)\end{array}$ & $\begin{array}{c}-0.48 \\
(-0.84,-0.12) \\
\mathbf{p}=\mathbf{0 . 0 0 9} \\
\end{array}$ & $\begin{array}{c}1.29 \\
(0.93)\end{array}$ & $\begin{array}{c}1.64 \\
(1.10)\end{array}$ & $\begin{array}{c}-0.37 \\
(-0.8,0.07) \\
p=0.096\end{array}$ \\
\hline $\begin{array}{c}\text { ACQ score } \\
\text { [7 questions] }\end{array}$ & $\begin{array}{c}1.88 \\
(1.00)\end{array}$ & $\begin{array}{c}1.96 \\
(0.79)\end{array}$ & $\begin{array}{c}1.50 \\
(0.67)\end{array}$ & $\begin{array}{c}1.90 \\
(0.90)\end{array}$ & $\begin{array}{c}-0.37 \\
(-0.67,-0.07) \\
\mathbf{p}=\mathbf{0 . 0 1 6}\end{array}$ & $\begin{array}{l}1.38 \\
(0.93)\end{array}$ & $\begin{array}{c}1.77 \\
(1.00)\end{array}$ & $\begin{array}{c}-0.35 \\
(-0.74,0.04) \\
p=0.081\end{array}$ \\
\hline $\begin{array}{c}\text { AQLQ } \\
\text { Total score }\end{array}$ & $\begin{array}{c}5.36 \\
(1.05)\end{array}$ & $\begin{array}{c}5.14 \\
(1.14)\end{array}$ & $\begin{array}{l}5.71 \\
(0.83)\end{array}$ & $\begin{array}{c}5.04 \\
(1.30)\end{array}$ & $\begin{array}{c}0.52 \\
(0.17,0.87) \\
\mathbf{p}=\mathbf{0 . 0 0 5}\end{array}$ & $\begin{array}{c}5.79 \\
(1.02)\end{array}$ & $\begin{array}{c}5.27 \\
(1.34)\end{array}$ & $\begin{array}{c}0.37 \\
(-0.02,0.76) \\
p=0.063\end{array}$ \\
\hline AQLQ Symptoms Domain & $\begin{array}{c}4.96 \\
(1.08)\end{array}$ & $\begin{array}{l}4.82 \\
(1.20)\end{array}$ & $\begin{array}{c}5.46 \\
(0.90)\end{array}$ & $\begin{array}{c}4.74 \\
(1.39)\end{array}$ & $\begin{array}{c}0.63 \\
(0.18,1.09) \\
\mathbf{p}=\mathbf{0 . 0 0 7}\end{array}$ & $\begin{array}{c}5.67 \\
(4.96,6.34)\end{array}$ & $\begin{array}{c}4.88 \\
(3.91,6.5)\end{array}$ & $\begin{array}{c}0.42 \\
(-0.05,0.89) \\
p=0.082\end{array}$ \\
\hline $\begin{array}{c}\text { AQLQ Activity Limitations } \\
\text { Domain }\end{array}$ & $\begin{array}{c}5.84 \\
(1.06)\end{array}$ & $\begin{array}{c}5.52 \\
(1.15)\end{array}$ & $\begin{array}{l}5.99 \\
(0.90)\end{array}$ & $\begin{array}{c}5.36 \\
(1.25)\end{array}$ & $\begin{array}{c}0.40 \\
(0.10,0.71) \\
\mathbf{p}=\mathbf{0 . 0 1 0}\end{array}$ & $\begin{array}{l}6.08 \\
(0.97)\end{array}$ & $\begin{array}{c}5.58 \\
(1.29)\end{array}$ & $\begin{array}{c}0.31 \\
(-0.01,0.63) \\
p=0.061\end{array}$ \\
\hline $\begin{array}{l}\text { AQLQ Emotional } \\
\text { Functions Domain }\end{array}$ & $\begin{array}{c}5.51 \\
(1.36)\end{array}$ & $\begin{array}{c}5.10 \\
(1.35)\end{array}$ & $\begin{array}{c}5.90 \\
(1.19)\end{array}$ & $\begin{array}{c}5.02 \\
(1.62)\end{array}$ & $\begin{array}{c}0.60 \\
(0.10,1.09) \\
\mathbf{p}=\mathbf{0 . 0 1 9}\end{array}$ & $\begin{array}{c}5.87 \\
(1.39)\end{array}$ & $\begin{array}{c}5.30 \\
(1.62)\end{array}$ & $\begin{array}{c}0.24 \\
(-0.26,0.74) \\
p=0.335\end{array}$ \\
\hline $\begin{array}{l}\text { AQLQ Environmental } \\
\text { Stimuli Domain }\end{array}$ & $\begin{array}{c}5.10 \\
(1.30)\end{array}$ & $\begin{array}{c}5.09 \\
(1.33)\end{array}$ & $\begin{array}{c}5.41 \\
(1.18)\end{array}$ & $\begin{array}{l}5.07 \\
(1.44)\end{array}$ & $\begin{array}{c}0.34 \\
(-0.09,0.77) \\
p=0.123\end{array}$ & $\begin{array}{c}5.71 \\
(1.15)\end{array}$ & $\begin{array}{c}5.13 \\
(1.47)\end{array}$ & $\begin{array}{c}0.55 \\
(0.07,1.03) \\
\mathbf{p}=\mathbf{0 . 0 2 6}\end{array}$ \\
\hline $\begin{array}{c}\text { Reliever inhaler puffs per } \\
24 \mathrm{hrs}\end{array}$ & $\begin{array}{c}3.19 \\
(2.55)\end{array}$ & $\begin{array}{l}2.94 \\
(2.93)\end{array}$ & $\begin{array}{l}2.58 \\
(2.67)\end{array}$ & $\begin{array}{c}3.97 \\
(5.45)\end{array}$ & $\begin{array}{c}-0.66 \\
(-1.69,0.37) \\
p=0.205\end{array}$ & $\begin{array}{l}2.13 \\
(2.63)\end{array}$ & $\begin{array}{c}1.99 \\
(2.33)\end{array}$ & $\begin{array}{c}-0.12 \\
(-1.35,1.11) \\
p=0.841\end{array}$ \\
\hline
\end{tabular}

Abbreviations: ACQ, Asthma Control Questionnaire; AQLQ, Asthma Quality of Life Questionnaire; PEF, peak expiratory flow rate; FEV ${ }_{1}$, forced expiratory volume in one second; Data represented as mean (SD) unless otherwise indicated.

${ }^{\dagger}$ Treatment difference from analysis of covariance, analysing change from baseline and adjusting for baseline value.

difference $-10.67 \mathrm{~L} / \mathrm{min}, 95 \% \mathrm{CI}-38.70$ to $17.37, \mathrm{p}=$ 0.449]. There was an improvement in the atorvastatin group compared to the placebo group at 4 weeks in the ACQ score [-0.37, 95\% CI -0.67 to $-0.07, \mathrm{p}=0.016]$ and AQLQ total score [0.52, 95\% CI 0.17 to $0.87 \mathrm{p}=0.005$ ] as well as AQLQ symptoms domain [0.63, 95\% CI 0.18 to $1.09 \mathrm{P}=0.007]$, activity limitations domain $[0.40,95 \% \mathrm{CI}$ 0.10 to $0.71 \mathrm{P}=0.010$ ] and emotional functions domain
[0.60, 95\% CI 0.10 to 1.09$) \mathrm{P}=0.019]$. At 8 weeks there was no significant improvement with atorvastatin and inhaled beclometasone compared to inhaled beclometasone alone in primary or exploratory outcome measures.

\section{Changes in inflammatory biomarkers}

After 4 and 8 weeks, the total cell count and differential cell counts in induced sputum were similar for 


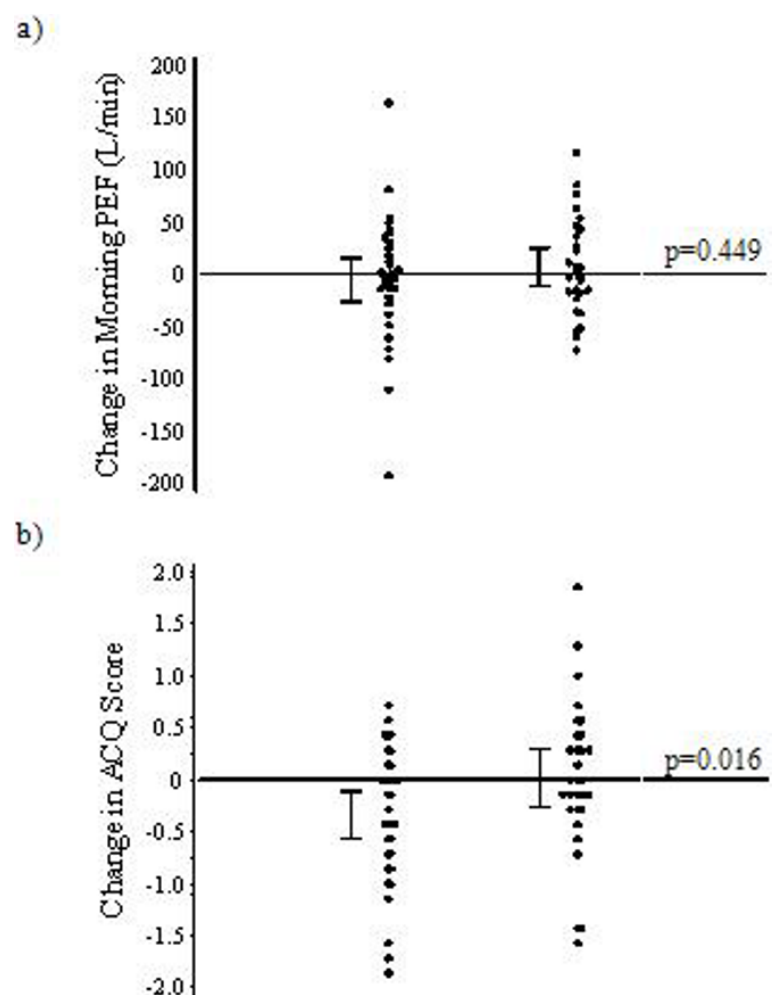

c)

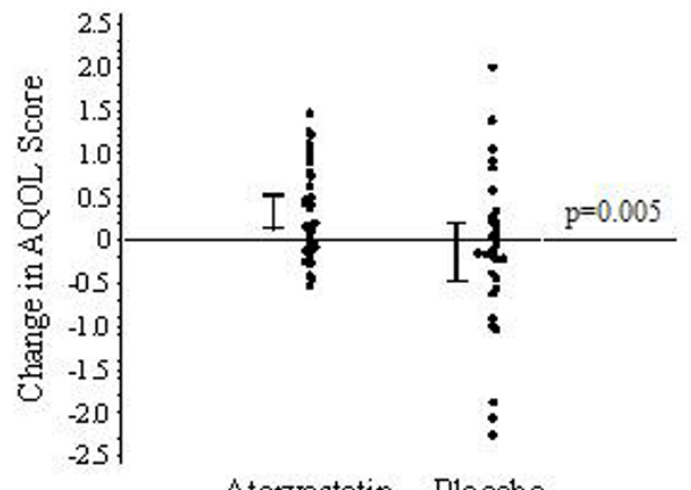

Atorvastatin Placebo

Figure 2 Change from baseline for both treatment groups in (a) morning PEF [L/min], (b) ACQ score, (c) AQLQ total score at 4 weeks. $P$ values are for comparisons of the mean change from baseline between the two treatments.

atorvastatin and placebo (Table 3). The sputum and serum concentrations of inflammatory cytokines and mediators were similar after atorvastatin compared to after placebo treatment at the 4 week and 8 week time points (Table 3 and data not shown). There was no significant change in $\mathrm{FE}_{\mathrm{NO}}$ after atorvastatin compared to placebo (data not shown).

\section{Change in biochemical markers}

The biochemical effects of atorvastatin therapy were reflected in significant reduction in concentration of serum lipids; cholesterol $[-1.55,95 \%$ CI -1.88 to -1.23 $\mathrm{P}<0.0001]$, LDL-cholesterol [-1.50, 95\% CI -1.88 to $-1.13 \mathrm{P}<0.0001]$ and triglyceride $[-0.49,95 \% \mathrm{CI}-0.87$, $-0.11 \mathrm{P}=0.013]$ (Table 4).

\section{Adverse events}

No suspected unexpected serious adverse reactions (SUSARs) were recorded. Adverse events (AEs) were similar between treatment groups. Two serious adverse events occurred, one in each group and neither related to study drug. For adverse events greater than $5 \%$ in frequency, the only severe $\mathrm{AE}$ was back pain in $3(8.3 \%)$ subjects in the atorvastatin group versus 0 in the placebo group. Moderate side effects were more frequent in the atorvastatin group: oral candidiasis ( 2 subjects versus 0 ), neck pain (2 versus 0 ) and asthma worsening (2 versus 1$)$.

\section{Compliance}

Compliance assessed by inhaler weight and tablet count was greater than $90 \%$ in both groups. The marked reduction in LDL-cholesterol in the group receiving atorvastatin (Table 4) provides further evidence of compliance. The weights of the returned inhalers indicate similar compliance with the inhaled beclometasone treatment.

\section{Discussion}

In this randomized controlled study we found no improvement in the atorvastatin group compared to the placebo group in lung function in smokers with mild to moderate asthma, whereas there was an improvement in ACQ and AQLQ scores at 4 weeks. Atorvastatin treatment had no anti-inflammatory effects on induced sputum cell counts or on selected sputum or circulating blood mediator levels.

Three previous randomized controlled crossover clinical trials reported an absence of clinical benefit with short-term statin therapy on asthma symptoms or lung function in non-smokers with asthma[9-11]. The lack of improvement in $\mathrm{PEF}, \mathrm{FEV}_{1}$ or airway responsiveness to methacholine in asthmatic smokers following atorvastatin supports the conclusions of these earlier studies that statins do not have a short-term beneficial effect on lung function in asthma irrespective of smoking status.

In addition to poor symptom control, smokers with asthma have worse asthma-specific quality of life scores [31]. The AQLQ is a well validated score of subjective well being and improving this index is an important goal of asthma management[32]. As one of a number of outcome measure we found a mean between-group difference in change from baseline in AQLQ score of 0.52 at 4 weeks, which is just greater than the minimal clinically significant change in score of $0.5[33]$. The 
Table 3 Inflammatory outcomes after atorvastatin treatment or placebo

\begin{tabular}{|c|c|c|c|c|c|c|c|c|}
\hline \multirow[t]{2}{*}{ Variables } & \multicolumn{2}{|c|}{ Baseline } & \multicolumn{2}{|c|}{4 weeks } & \multirow{2}{*}{$\begin{array}{l}\text { Treatment difference, } \\
\text { atorvastatin minus } \\
\text { placebo }(95 \% \mathrm{Cl})\end{array}$} & \multicolumn{2}{|c|}{8 weeks } & \multirow{2}{*}{$\begin{array}{l}\text { Treatment difference, } \\
\text { atorvastatin minus } \\
\text { placebo }(95 \% \mathrm{Cl})\end{array}$} \\
\hline & $\begin{array}{l}\text { Atorvastatin } \\
(\mathrm{n}=35)\end{array}$ & $\begin{array}{l}\text { Placebo } \\
(\mathrm{n}=32)\end{array}$ & $\begin{array}{c}\text { Atorvastatin } \\
(\mathrm{n}=31)\end{array}$ & $\begin{array}{l}\text { Placebo } \\
(\mathrm{n}=29)\end{array}$ & & $\begin{array}{c}\text { Atorvastatin \& ICS } \\
(\mathrm{n}=32)\end{array}$ & $\begin{array}{c}\text { Placebo \& ICS } \\
\quad(n=24)\end{array}$ & \\
\hline \multicolumn{9}{|l|}{$\begin{array}{c}\text { Sputum differential } \\
\text { cell count } \& \\
\text { proportion }(\%)\end{array}$} \\
\hline Total cell count $\left(\times 10^{4}\right)$ & $\begin{array}{c}651.5 \\
(547.0,890.5)\end{array}$ & $\begin{array}{c}620.8 \\
(543.2,758.8)\end{array}$ & $\begin{array}{c}628.5 \\
(533.5,921.5)\end{array}$ & $\begin{array}{c}602.0 \\
(512.0,791.0)\end{array}$ & $\begin{array}{c}43.0 \\
(-75.2,161.1) \\
p=0.469\end{array}$ & $\begin{array}{c}607.8 \\
(517.0,785.5)\end{array}$ & $\begin{array}{c}658.5 \\
(563.2,860.2)\end{array}$ & $\begin{array}{c}-81.6 \\
(-177.2,14.0) \\
p=0.093\end{array}$ \\
\hline $\begin{array}{c}\text { Macrophage }\left(\times 10^{4}\right) \\
\text { Macrophage } \%\end{array}$ & $\begin{array}{c}154.5 \\
(111.5,263.0) \\
38.4 \\
(29.6,61.3)\end{array}$ & $\begin{array}{c}179.8 \\
(87.2,242.2) \\
45.1 \\
(31.8,58.7)\end{array}$ & $\begin{array}{c}159.5 \\
(132.0,221.0) \\
45.5 \\
(31.5,58.9)\end{array}$ & $\begin{array}{c}142.5 \\
(81.0,220.0) \\
38.5 \\
(24.6,54.6)\end{array}$ & $\begin{array}{c}16.9 \\
(-27.5,61.3) \\
p=0.450 \\
4.8 \\
(-5.9,15.5) \\
p=0.374\end{array}$ & $\begin{array}{c}173.2 \\
(126.2,247.2) \\
41.4 \\
(34.1,54.9)\end{array}$ & $\begin{array}{c}174.2 \\
(122.8,250.5) \\
44.2 \\
(32.3,59.0)\end{array}$ & $\begin{array}{c}-3.0 \\
(-47.6,41.6) \\
p=0.893 \\
-0.5 \\
(-9.6,8.6) \\
p=0.913\end{array}$ \\
\hline $\begin{array}{c}\text { Neutrophil }\left(\times 10^{4}\right) \\
\text { Neutrophil \% }\end{array}$ & $\begin{array}{c}131.0 \\
(76.0,232.5) \\
37.8 \\
(18.3,54.2)\end{array}$ & $\begin{array}{c}122.8 \\
(56.2,201.2 \\
29.9 \\
(15.0,49.1)\end{array}$ & $\begin{array}{c}136.5 \\
(61.0,233.5) \\
33.6 \\
(18.2,55.8)\end{array}$ & $\begin{array}{c}99.0 \\
(69.5,299.5) \\
31.5 \\
(17.6,63.2)\end{array}$ & $\begin{array}{c}-19.2 \\
(-72.0,33.6) \\
p=0.470 \\
-4.7 \\
(-15.7,6.4) \\
p=0.401\end{array}$ & $\begin{array}{c}151.8 \\
(80.5,211.5) \\
38.3 \\
(23.0,52.8)\end{array}$ & $\begin{array}{c}149.0 \\
(78.2,229.0) \\
34.9 \\
(20.7,49.5)\end{array}$ & $\begin{array}{c}-8.0 \\
(-49.4,33.4) \\
p=0.700 \\
2.1 \\
(-8.0,12.1) \\
p=0.680\end{array}$ \\
\hline $\begin{array}{c}\text { Eosinophil }\left(\times 10^{4}\right) \\
\text { Eosinophil } \%\end{array}$ & $\begin{array}{c}3.0 \\
(0.5,7.5) \\
0.6 \\
(0.1,2.4)\end{array}$ & $\begin{array}{c}1.5 \\
(0.0,5.8) \\
0.4 \\
(0.0,1.4)\end{array}$ & $\begin{array}{c}1.5 \\
(0.0,4.5) \\
0.4 \\
(0.0,1.0)\end{array}$ & $\begin{array}{c}1.5 \\
(0.0,3.5) \\
0.3 \\
(0.0,1.2)\end{array}$ & $\begin{array}{c}-3.2(-8.6,2.3) \\
p=0.253 \\
-1.0 \\
(-2.3,0.3) \\
p=0.138\end{array}$ & $\begin{array}{c}1.3 \\
(0.3,5.5) \\
0.4 \\
(0.1,1.1)\end{array}$ & $\begin{array}{c}0.8 \\
(0,2.5) \\
0.2 \\
(0,0.6)\end{array}$ & $\begin{array}{c}0.4 \\
(-1.6,2.5) \\
p=0.677 \\
0.1 \\
(-0.4,0.5) \\
p=0.798\end{array}$ \\
\hline $\begin{array}{c}\text { Lymphocyte }\left(\times 10^{4}\right) \\
\text { Lymphocyte } \%\end{array}$ & $\begin{array}{c}1.5 \\
(0.0,3.0) \\
0.3 \\
(0.0,0.7)\end{array}$ & $\begin{array}{c}0.8 \\
(0.0,2.8) \\
0.2 \\
(0.0,0.7)\end{array}$ & $\begin{array}{c}0.5 \\
(0.0,3) \\
0.1 \\
(0.0,0.7)\end{array}$ & $\begin{array}{c}0.5 \\
(0.0,1.5) \\
0.1 \\
(0.0,0.5)\end{array}$ & $\begin{array}{c}0.8 \\
(-0.2,1.9) \\
p=0.125 \\
0.1 \\
(-0.2,0.4) \\
p=0.425\end{array}$ & $\begin{array}{c}0.8 \\
(0.0,2.0) \\
0.2 \\
(0.0,0.7)\end{array}$ & $\begin{array}{c}1.3 \\
(0.0,2.5) \\
0.4 \\
(0.0,0.6)\end{array}$ & $\begin{array}{c}-0.1 \\
(-1.3,1.1) \\
p=0.865 \\
0.1 \\
(-0.3,0.4) \\
p=0.757\end{array}$ \\
\hline $\begin{array}{c}\text { Bronchial epithelial cell }\left(\times 10^{4}\right) \\
\text { Br. epithelial cell } \%\end{array}$ & $\begin{array}{c}44.0 \\
(26.0,90.5) \\
11.5 \\
(5.9,26.0)\end{array}$ & $\begin{array}{c}49.5 \\
(29.0,117.0) \\
12.3 \\
(7.8,29.2)\end{array}$ & $\begin{array}{c}33.0 \\
(18.0,78.0) \\
8.4 \\
(5.9,17.8)\end{array}$ & $\begin{array}{c}37.5 \\
(24.0,89.5) \\
9.2 \\
(5.6,24.8)\end{array}$ & $\begin{array}{c}20.6 \\
(-9.6,50.9) \\
p=0.177 \\
1.6 \\
(-5.9,9.0) \\
p=0.680\end{array}$ & $\begin{array}{c}40.0 \\
(28.0,75.8) \\
11.2 \\
(6.8,22.2)\end{array}$ & $\begin{array}{c}57.8 \\
(39.5,92.8) \\
13.3 \\
(9.2,22.5)\end{array}$ & $\begin{array}{c}-10.3 \\
(-32.7,12.1) \\
p=0.361 \\
-1.3 \\
(-7.7,5.1) \\
p=0.679\end{array}$ \\
\hline
\end{tabular}


Table 3 Inflammatory outcomes after atorvastatin treatment or placebo (Continued)

\begin{tabular}{|c|c|c|c|c|c|c|c|c|}
\hline Sputum mediators & & & & & & & & \\
\hline $\begin{array}{l}\text { Leukotriene } B_{4} \\
(\mathrm{pg} / \mathrm{ml})\end{array}$ & $\begin{array}{c}103.1 \\
(79.7,185.4)\end{array}$ & $\begin{array}{c}172.0 \\
(106.0,422.4)\end{array}$ & $\begin{array}{c}98.4 \\
(56.9,215.2)\end{array}$ & $\begin{array}{c}175.2 \\
(77.0,336.0)\end{array}$ & $\begin{array}{c}-49.4 \\
(-205.1,106.4) \\
p=0.528\end{array}$ & $\begin{array}{c}104.9 \\
(41.9,151.5)\end{array}$ & $\begin{array}{c}159.0 \\
(81.0,520.2)\end{array}$ & $\begin{array}{c}-231.4 \\
(-589.5,126.6) \\
p=0.200\end{array}$ \\
\hline $\begin{array}{c}\text { MPO } \\
\text { (ng/mL) }\end{array}$ & $\begin{array}{c}16.5 \\
(6.4,39.6)\end{array}$ & $\begin{array}{c}27.0 \\
(10.6,40.6)\end{array}$ & $\begin{array}{c}16.4 \\
(9.0,30.5)\end{array}$ & $\begin{array}{c}20.6 \\
(10.6,34.1)\end{array}$ & $\begin{array}{c}-16.5 \\
(-48.0,15.0) \\
p=0.298\end{array}$ & $\begin{array}{c}14.8 \\
(6.4,34.5)\end{array}$ & $\begin{array}{c}28.1 \\
(8.9,68.8)\end{array}$ & $\begin{array}{c}-111.2 \\
(-264.8,42.5) \\
p=0.152\end{array}$ \\
\hline Interleukin-6 (pg/ml) & $\begin{array}{c}139.5 \\
(33.9,276.5)\end{array}$ & $\begin{array}{c}220.4 \\
(70.0,498.5)\end{array}$ & $\begin{array}{c}109.3 \\
(33.9,351.5)\end{array}$ & $\begin{array}{c}144.4 \\
(40.3,391.6)\end{array}$ & $\begin{array}{c}-5.6 \\
(-254.1,243.0) \\
p=0.964\end{array}$ & $\begin{array}{c}147.2 \\
(35.9,223.0)\end{array}$ & $\begin{array}{c}122.0 \\
(34.1,518.6)\end{array}$ & $\begin{array}{c}-22.5 \\
(-247.3,202.3) \\
p=0.841\end{array}$ \\
\hline Interleukin-8 (pg/ml) & $\begin{array}{c}813.1 \\
(238.8,3000.0)\end{array}$ & $\begin{array}{c}3000.0 \\
(426.1,3000.0)\end{array}$ & $\begin{array}{c}408.5 \\
(179.7,3000.0)\end{array}$ & $\begin{array}{c}1686.0 \\
(353.2,3000.0)\end{array}$ & $\begin{array}{c}-453.4 \\
(-1071.9,165.1) \\
p=0.127\end{array}$ & $\begin{array}{c}386.5 \\
(180.7,3000.0)\end{array}$ & $\begin{array}{c}541.5 \\
(239.4,3000.0)\end{array}$ & $\begin{array}{c}172.3 \\
(-600.8,945.4) \\
p=0.656\end{array}$ \\
\hline Interleukin-10 (pg/ml) & $\begin{array}{c}13.6 \\
(1.0,126.9)\end{array}$ & $\begin{array}{c}13.0 \\
(1.2,80.9)\end{array}$ & $\begin{array}{c}1.8 \\
(0.3,105.3)\end{array}$ & $\begin{array}{c}15.4 \\
(0.8,53.4)\end{array}$ & $\begin{array}{c}27.5 \\
(-53.2,108.2) \\
p=0.497\end{array}$ & $\begin{array}{c}6.1 \\
(0.7,72.6)\end{array}$ & $\begin{array}{c}4.6 \\
(0.7,62.9)\end{array}$ & $\begin{array}{c}-4.2 \\
(-132.8,124.5) \\
p=0.948\end{array}$ \\
\hline \multicolumn{9}{|l|}{ Serum biomarkers } \\
\hline hs-CRP (mg/L) & $\begin{array}{c}2.8 \\
(1.6,4.0)\end{array}$ & $\begin{array}{c}3.6 \\
(1.5,6.9)\end{array}$ & $\begin{array}{c}2.6 \\
(1.6,5.0)\end{array}$ & $\begin{array}{c}2.8 \\
(1.4,5.6)\end{array}$ & $\begin{array}{c}0.9 \\
(-4.1,5.9) \\
p=0.718\end{array}$ & $\begin{array}{c}1.9 \\
(1.0,4.1)\end{array}$ & $\begin{array}{c}2.1 \\
(1.0,5.1)\end{array}$ & $\begin{array}{c}0.6 \\
(-2.8,3.9) \\
p=0.731\end{array}$ \\
\hline Interleukin-6 (pg/ml) & $\begin{array}{c}2.0 \\
(0.2,4.1)\end{array}$ & $\begin{array}{c}2.6 \\
(0.2,30.0)\end{array}$ & $\begin{array}{c}2.7 \\
(0.2,11.0)\end{array}$ & $\begin{array}{c}1.8 \\
(0.2,26.0)\end{array}$ & $\begin{array}{c}-9.1 \\
(-20.8,2.7) \\
p=0.127\end{array}$ & $\begin{array}{c}0.4 \\
(0.2,8.8)\end{array}$ & $\begin{array}{c}3.1 \\
(0.2,21.2)\end{array}$ & $\begin{array}{c}16.7 \\
(-29.5,62.8) \\
p=0.472\end{array}$ \\
\hline
\end{tabular}

Abbreviations: CRP, C-reactive protein; MPO, myeloperoxisade; Mediator levels pg/ml unless otherwise indicated. Data represented as median (IQR) unless otherwise indicated. 
Table 4 Serum biochemical outcomes after atorvastatin treatment or placebo

\begin{tabular}{|c|c|c|c|c|c|}
\hline \multirow[t]{2}{*}{ Variables } & \multicolumn{2}{|c|}{ Baseline } & \multicolumn{2}{|c|}{8 weeks } & \multirow{2}{*}{$\begin{array}{l}\text { Treatment difference, } \\
\text { atorvastatin minus } \\
\text { placebo }(95 \% \mathrm{Cl})\end{array}$} \\
\hline & $\begin{array}{l}\text { Atorvastatin } \\
(\mathrm{n}=35)\end{array}$ & $\begin{array}{l}\text { Placebo } \\
(\mathrm{n}=32)\end{array}$ & $\begin{array}{c}\text { Atorvastatin \& ICS } \\
(\mathrm{n}=32)\end{array}$ & $\begin{array}{l}\text { Placebo \&ICS } \\
(\mathrm{n}=25)\end{array}$ & \\
\hline $\begin{array}{c}\text { Total cholesterol } \\
(\mathrm{mmol} / \mathrm{l})\end{array}$ & $\begin{array}{c}5.15 \\
(0.89)\end{array}$ & $\begin{array}{l}5.67 \\
(1.12)\end{array}$ & $\begin{array}{c}3.62 \\
(0.76)\end{array}$ & $\begin{array}{l}5.34 \\
(0.90)\end{array}$ & $\begin{array}{c}-1.55 \\
(-1.88,-1.23) \\
P<0.0001\end{array}$ \\
\hline $\begin{array}{l}\text { Triglycerides } \\
\text { (mmol/l) }\end{array}$ & $\begin{array}{c}1.59 \\
(0.87)\end{array}$ & $\begin{array}{c}2.18 \\
(1.41)\end{array}$ & $\begin{array}{c}1.02 \\
(0.35)\end{array}$ & $\begin{array}{c}1.74 \\
(1.29)\end{array}$ & $\begin{array}{c}-0.49 \\
(-0.87,-0.11) \\
\mathbf{P}=\mathbf{0 . 0 1 3}\end{array}$ \\
\hline $\begin{array}{l}\text { LDL-cholesterol } \\
\text { (mmol/l) }\end{array}$ & $\begin{array}{c}3.19 \\
(0.82)\end{array}$ & $\begin{array}{c}3.49 \\
(0.99)\end{array}$ & $\begin{array}{c}1.89 \\
(0.62)\end{array}$ & $\begin{array}{l}3.46 \\
(0.87)\end{array}$ & $\begin{array}{c}-1.50 \\
(-1.88,-1.13) \\
P<0.0001\end{array}$ \\
\hline $\begin{array}{l}\text { HDL-cholesterol } \\
\text { (mmol/l) }\end{array}$ & $\begin{array}{c}1.24 \\
(0.35)\end{array}$ & $\begin{array}{l}1.26 \\
(0.31)\end{array}$ & $\begin{array}{c}1.29 \\
(0.35)\end{array}$ & $\begin{array}{l}1.23 \\
(0.27)\end{array}$ & $\begin{array}{c}0.03 \\
(-0.06,0.13) \\
p=0.497\end{array}$ \\
\hline $\begin{array}{l}\text { Alkaline phosphatase } \\
\text { (IU/I) }\end{array}$ & $\begin{array}{l}83.33 \\
(31.36)\end{array}$ & $\begin{array}{l}75.16 \\
(21.04)\end{array}$ & $\begin{array}{c}98.62 \\
(91.26)\end{array}$ & $\begin{array}{c}77.16 \\
(19.58)\end{array}$ & $\begin{array}{c}5.94 \\
(-15.67,27.54) \\
p=0.584\end{array}$ \\
\hline $\begin{array}{l}\text { ALT } \\
\text { (IU/I) }\end{array}$ & $\begin{array}{l}17.58 \\
(9.27)\end{array}$ & $\begin{array}{l}22.50 \\
(11.88)\end{array}$ & $\begin{array}{l}22.00 \\
(14.37)\end{array}$ & $\begin{array}{l}24.04 \\
(11.70)\end{array}$ & $\begin{array}{c}3.67 \\
(-1.84,9.18) \\
p=0.188\end{array}$ \\
\hline
\end{tabular}

Abbreviations: ALT, alanine aminotransferase.

Data represented as mean (SD).

individual AQLQ domains for symptoms and emotions also crossed this threshold. The improvement in AQLQ score with atorvastatin is comparable to that reported with long-acting $\beta_{2}$ agonist therapy[34] and with leukotriene receptor antagonist therapy in non-smokers with asthma[35]. The non-significant mean improvement in ACQ score of -0.37 did not reach the minimum important reduction threshold of $-0.5[36]$. Improvements in subject-centered outcomes with interventions in asthma are usually associated with better lung function, although this is not always the case. For example, clinically important improvements in the AQLQ score were observed in a trial comparing different formulations of inhaled beclometasone[37] and in a double-blind shamcontrolled trial of bronchial thermoplasty[38] while in both studies conventional clinical indices of lung function and asthma control were similar.

Statins restore corticosteroid sensitivity in patients with asthma[14,15], possibly by restoring HDAC activity [16] or by increased induction of indoleamine 2, 3-dioxygense resulting in increased secretion of the antiinflammatory cytokine IL-10[15]. Statins also inhibit IL17 expression and secretion from human Th17 cells[39], which have been implicated in causing corticosteroid resistance[40]. Based on these studies we wished to determine whether atorvastatin might restore sensitivity to corticosteroids and had hoped to observe a beneficial effect of atorvastatin and inhaled beclometasone compared to inhaled beclometasone alone, but found no significant effect in outcome measures at the 8 week time point. It is possible that 4 weeks was not long enough to demonstrate a synergistic effect, although in previous studies a 4 week treatment period was long enough to show the potentiating effects of low dose theophylline on corticosteroid sensitivity in smokers with asthma[41].

Statins have pleiotropic properties including antiinflammatory and immuno-modulatory effects [5] that may be beneficial in the treatment of chronic inflammatory airway diseases[6]. The anti-inflammatory effects of statins are thought to be mediated by inhibition of the isoprenylation of small G-protein signalling molecules and by prevention of lipid raft formation, pathways involved in cell proliferation, activation and oxidative stress. Statins have immune modulating properties in animal models of allergic asthma[7,8] and COPD[12,13]. In our study, a limited investigation of circulating and airway inflammatory cells and mediators failed to show any effects of atorvastatin therapy on inflammatory biomarkers including CRP, despite good drug compliance and significant reduction of serum lipid markers.

There are several limitations of the study. Firstly, if fewer than three days of data were recorded during the seven days immediately preceding each study visit, patient diary entries for morning PEF were substituted in place of the missing value, which raises the possibility of increased variability in PEF measurements. However this procedure was performed in a minority of PEF 
measurements used in the analysis and is unlikely to have influenced the PEF result. Secondly, while questionnaire scores were not significantly different at baseline, it is possible that the slight difference in the proportion of participants taking LABA prior to screening may have had an effect on subsequent measurements of asthma control. The short duration of treatment with atorvastatin may have missed an effect on clinical or inflammatory outcomes. Future studies should assess whether treatment with statins influence outcome measures such as exacerbation rate and decline in lung function in smokers with asthma and in individuals with more severe disease. Statins have an inhibitory effect on human airway smooth muscle cell proliferation [42] and indices of airway remodelling [43-45]. It is possible that the administration of atorvastatin therapy for a longer duration of time may improve outcome measures of airway remodelling in asthma'

\section{Conclusion}

In conclusion, short-term treatment with atorvastatin does not improve lung function in smokers with mild to moderate asthma, but may improve asthma quality of life. Further trials using statins in asthmatic smokers are indicated.

\section{Acknowledgements}

The Medical Research Council UK funded the clinical trial; study medication was provided by Pfizer (atorvastatin) and Trinity-Chiesi (Clenil Modulite inhalers). We would also like to thank the Scottish Primary Care Research Network, the participants and the participating general practices from the Greater Glasgow and Lanarkshire region. Without their help this study would not have been possible.

\section{Author details}

${ }^{1}$ Respiratory Medicine, Institute of Infection, Immunity \& Inflammation, University of Glasgow, Glasgow, UK. ${ }^{2}$ Immunology, Institute of Infection, Immunity \& Inflammation, University of Glasgow, Glasgow, UK. ${ }^{3}$ MRC Hub for Trials Methodology Research, University of Edinburgh, UK. ${ }^{4}$ Robertson Centre for Biostatistics, University of Glasgow, Glasgow, UK. ${ }^{5}$ General Practice, University of Glasgow, Glasgow, UK.

\section{Authors' contributions}

GB: Contributed to the acquisition and interpretation of the data; RC: Contributed to the acquisition and interpretation of the data and drafted the manuscript for important intellectual content; CMcS: Contributed interpretation of the data, carried out the immunoassays and drafted the manuscript for important intellectual content; MB: Contributed to the acquisition of the data; CW: Participated in the design of the study and performed the statistical analysis; ID: Carried out the immunoassays; LJ: Carried out the immunoassays; $J L$ : Contributed to the acquisition of the data; SL: Performed the statistical analysis; MS: Contributed to interpretation of the data and drafted the manuscript for important intellectual content; FM: Contributed to the acquisition and interpretation of the data; NCT: Conceived the study, participated in its design, contributed to interpretation of the data and drafted the manuscript for important intellectual content. All authors read and approved the final manuscript.

\section{Competing interests}

The authors declare that they have no competing interests.
Received: 24 November 2010 Accepted: 7 April 2011

Published: 7 April 2011

\section{References}

1. Chaudhuri R, Livingston E, McMahon AD, Thomson L, Borland W, Thomson NC: Cigarette smoking impairs the therapeutic response to oral corticosteroids in chronic asthma. Am J Respir Crit Care Med 2003, 168(11):1308-1311.

2. Chalmers GW, Macleod KJ, Little SA, Thomson LJ, McSharry CP, Thomson NC: Influence of cigarette smoking on inhaled corticosteroid treatment in mild asthma. Thorax 2002, 57(3):226-230.

3. Tomlinson JEM, MCMahon AD, Chaudhuri R, Thompson JM, Wood SF, Thomson NC: Efficacy of low and high dose inhaled corticosteroid in smokers versus non-smokers with mild asthma. Thorax 2005, 60(4):282-287.

4. Thomson N, Chaudhuri R: Asthma in smokers: challenges and opportunities. Curr Opin Pulm Med 2009, 15:39-45.

5. Greenwood J, Steinman L, Zamvil SS: Statin therapy and autoimmune disease: from protein prenylation to immunomodulation. Nat Rev Immunol 2006, 6(5):358-370.

6. Hothersall E, McSharry C, Thomson NC: Potential therapeutic role for statins in respiratory disease. Thorax 2006, 61(8):729-734.

7. McKay A, Leung BP, Mclnnes IB, Thomson NC, Liew FY: A Novel AntiInflammatory Role of Simvastatin in a Murine Model of Allergic Asthma. $\mathrm{J}$ Immunol 2004, 172(5):2903-2908.

8. Zeki AA, Franzi L, Last J, Kenyon NJ: Simvastatin Inhibits Airway Hyperreactivity: Implications for the Mevalonate Pathway and Beyond. Am J Respir Crit Care Med 2009, 180(8):731-740.

9. Menzies D, Nair A, Meldrum KT, Fleming D, Barnes M, Lipworth BJ: Simvastatin does not exhibit therapeutic anti-inflammatory effects in asthma. J Allergy Clin Immunol 2007, 119(2):328-335.

10. Hothersall EJ, Chaudhuri R, McSharry C, Donnelly I, Lafferty J, McMahon AD, Weir CJ, Meiklejohn J, Sattar N, Mclnnes I, et al: Effects of atorvastatin added to inhaled corticosteroids on lung function and sputum cell counts in atopic asthma. Thorax 2008, 63(12):1070-1075.

11. Cowan DC, Cowan JO, Palmay R, Williamson A, Taylor DR: Simvastatin in the treatment of asthma: lack of steroid-sparing effect. Thorax 2010, 65(10):891-896.

12. Lee J-H, Lee D-S, Kim E-K, Choe K-H, Oh Y-M, Shim T-S, Kim S-E, Lee Y-S, Lee S-D: Simvastatin Inhibits Cigarette Smoking-induced Emphysema and Pulmonary Hypertension in Rat Lungs. Am J Respir Crit Care Med 2005, 172(8):987-993.

13. Takahashi S, Nakamura $H$, Seki M, Shiraishi $Y$, Yamamoto M, Furuuchi M, Nakajima T, Tsujimura S, Shirahata T, Nakamura M, et al: Reversal of elastase-induced pulmonary emphysema and promotion of alveolar epithelial cell proliferation by simvastatin in mice. Am J Physiol 2008, 294(5):L882-890

14. Samson K, Minoguchi K, Tanaka A, Oda N, Yokoe T, Yamamoto Y, Yamamoto M, Ohta S, Adachi M: Inhibitory effects of fluvastatin on cytokine and chemokine production by peripheral blood mononuclear cells in patients with allergic asthma. Clin Exp Allergy 2006, 36(4):475-482

15. Maneechotesuwan K, Ekjiratrakul W, Kasetsinsombat K, Wongkajornsilp A, Barnes PJ: Statins enhance the anti-inflammatory effects of inhaled corticosteroids in asthmatic patients through increased induction of indoleamine 2, 3-dioxygenase. J Allergy Clin Immunol 2010, 126(4):754-762.

16. N'Guessan PD, Riediger F, Vardarova K, Scharf S, Eitel J, Opitz B, Slevogt H, Weichert W, Hocke AC, Schmeck B, et al: Statins Control Oxidized LDLMediated Histone Modifications and Gene Expression in Cultured Human Endothelial Cells. Arterioscler Thromb Vasc Biol 2009, 29(3):380-386.

17. Ito K, Lim S, Caramori G, Chung K, Barnes P, Adcock I: Cigarette smoking reduces histone deacetylase 2 expression, enhances cytokine expression, and inhibits glucocorticoid actions in alveolar macrophages. FASEB $J$ 2001, 15(6):1110-1112.

18. Alexeeff SE, Litonjua AA, Sparrow D, Vokonas PS, Schwartz J: Statin Use Reduces Decline in Lung Function: VA Normative Aging Study. Am J Respir Crit Care Med 2007, 176(8):742-747.

19. Janda S, Park K, FitzGerald JM, Etminan M, Swiston J: Statins in COPD. Chest 2009, 136(3):734-743.

20. Joukhadar C, Klein N, Prinz M, Schrolnberger C, Vukovich T, Wolzt M, Schemetterer L, Dorner GT: Similar effects of atorvastatin, simvastatin and 
pravastatin on thrombogenic and inflammatory parameters in patients with hypercholesterolaemia. Thombosis and Haemostasis 2001, 85:47-51.

21. McCarey DW, Mclnnes IB, Madhok R, Hampson R, Scherbakova O, Ford I, Capell HA, Sattar N: Trial of Atorvastatin in Rheumatoid Arthritis (TARA): double-blind, randomised placebo-controlled trial. Lancet 2004, 363:2015-2021.

22. Chan KY, Boucher ES, Gandhi PJ, Silva MA: HMG-CoA reductase inhibitors for lowering elevated levels of C-reactive protein. American Journal of Health-System Pharmacy 2004, 61(16):1676-1681.

23. GINA Report, Global Strategy for Asthma Management and Prevention. [http://www.ginasthma.com].

24. Santanello N, Barber B, Reiss T, Friedman B, Juniper E, Zhang J: Measurement characteristics of two asthma symptom diary scales for use in clinical trials. Eur Respir J 1997, 10:646-651.

25. Juniper EF, O'Byrne PM, Guyatt GH, Ferrie PJ, King DR: Development and validation of a questionnaire to measure asthma control. Eur Respir J 1999, 14(4):902-907.

26. Juniper EF, Guyatt GH, Cox FM, Ferrie PJ, King DR: Development and validation of the Mini Asthma Quality of Life Questionnaire. Eur Respir $J$ 1999, 14(1):32-38.

27. ATS/ERS Recommendations for Standardized Procedures for the Online and Offline Measurement of Exhaled Lower Respiratory Nitric Oxide and Nasal Nitric Oxide. Am J Respir Crit Care Med 2005, 171(8):912-930.

28. Cockcroft D, Killian D, Mellon J, Hargreave F: Bronchial reactivity to inhaled histamine: a method and clinical survey. Clin Allergy 1977, 7:235-243.

29. Pavord I, Pizzichini M, Pizzichini E, Hargreave F: The use of induced sputum to investigate airway inflammation. Thorax 1997, 52(6):498-501.

30. Greening AP, Ind PW, Northfield M, Shaw G: Added salmeterol versus higher-dose corticosteroid in asthma patients with symptoms on existing inhaled corticosteroid. The Lancet 1994, 344(8917):219-224.

31. Eisner MD, Iribarren $C$ : The influence of cigarette smoking on adult asthma outcomes. Nicotine Tob Res 2007, 9(1):53-56.

32. Siroux V, Boudier A, Anto JM, Cazzoletti L, Accordini S, Alonso J, Cerveri I, Corsico A, Gulsvik A, Jarvis D, et al: Quality-of-life and asthma-severity in general population asthmatics: results of the ECRHS II study. Allergy 2008, 63(5):547-554.

33. Juniper EF, Guyatt GH, Willan A, Griffith LE: Determining a minimal important change in a disease-specific quality of life questionnaire. J Clin Epidemiol 1994, 47(1):81-87.

34. Juniper E, Johnston P, Borkhoff C, Guyatt G, Boulet L, Haukioja A: Quality of life in asthma clinical trials: comparison of salmeterol and salbutamol. Am J Respir Crit Care Med 1995, 151(1):66-70.

35. Dahlen S-E, Malmstrom K, Nizankowska EWA, Dahlen B, Kuna P, Kowalski M, Lumry WR, Picado C, Stevenson DD, Bousquet J, et al: Improvement of Aspirin-Intolerant Asthma by Montelukast, a Leukotriene Antagonist. A Randomized, Double-Blind, Placebo-Controlled Trial. Am J Repir Crit Care Med 2002, 165(1):9-14.

36. Juniper E, Svensson K, Mork A, Stahl E: Measurement properties and interpretation of three shortened versions of the asthma control questionnaire. Resp Med 2005, 99:553-558.

37. Juniper E, Price D, Stampone P, Creemers J, Mol S, Fireman P: Clinically Important Improvements in Asthma-Specific Quality of Life, But No Difference in Conventional Clinical Indexes in Patients Changed From Conventional Beclomethasone Dipropionate to Approximately Half the Dose of Extrafine Beclomethasone Dipropionate*. Chest 2002, 121(6):1824-1832

38. Castro M, Rubin AS, Laviolette M, Fiterman J, De Andrade Lima M, Shah PL, Fiss E, Olivenstein R, Thomson NC, Niven RM, et al: Effectiveness and Safety of Bronchial Thermoplasty in the Treatment of Severe Asthma: A Multicenter, Randomized, Double-Blind, Sham-Controlled Clinical Trial. Am J Respir Crit Care Med 2010, 181(2):116-124.

39. Zhang X, Jin J, Peng X, Ramgolam VS, Markovic-Plese S: Simvastatin Inhibits IL-17 Secretion by Targeting Multiple IL-17-Regulatory Cytokines and by Inhibiting the Expression of IL-17 Transcription Factor RORC in CD4+ Lymphocytes. J Immunol 2008, 180(10):6988-6996.

40. McKinley L, Alcorn JF, Peterson A, DuPont RB, Kapadia S, Logar A, Henry A, Irvin CG, Piganelli JD, Ray A, et al: TH17 Cells Mediate Steroid-Resistant Airway Inflammation and Airway Hyperresponsiveness in Mice. $J$ Immunol 2008, 181(6):4089-4097.

41. Spears M, Donnelly I, Jolly L, Brannigan M, Ito K, McSharry C, Lafferty J, Chaudhuri R, Braganza G, Adcock IM, et al: Effect of low-dose theophylline plus beclometasone on lung function in smokers with asthma: a pilot study. Eur Respir J 2009, 33(5):1010-1017.

42. Takeda N, Kondo M, Ito S, Ito Y, Shimokata K, Kume H: Role of RhoA Inactivation in Reduced Cell Proliferation of Human Airway Smooth Muscle by Simvastatin. Am J Respir Cell Mol Biol 2006, 35(6):722-729.

43. Wright JL, Zhou S, Preobrazhenska O, Marshall C, Sin DD, Laher I, Golbidi S, Churg AM: Statin Reverses Smoke-induced Pulmonary Hypertension and Prevents Emphysema but Not Airway Remodeling. Am J Respir Crit Care Med 2011, 183(1):50-58.

44. Zeki AA, Bratt JM, Rabowsky M, Last JA, Kenyon NJ: Simvastatin inhibits goblet cell hyperplasia and lung arginase in a mouse model of allergic asthma: a novel treatment for airway remodeling? Translational Research 2010, 156(6):335-349.

45. Ou X, Wen F, Uhal B, Feng Y, Huang X, Wang T, Wang K, Liu D, Wang X, Chen $L$ : Simvastatin attenuates experimental small airway remodelling in rats. Respirology 2009, 14(5):734-745.

\section{Pre-publication history}

The pre-publication history for this paper can be accessed here: http://www.biomedcentral.com/1471-2466/11/16/prepub

doi:10.1186/1471-2466-11-16

Cite this article as: Braganza et al:: Effects of short-term treatment with atorvastatin in smokers with asthma - a randomized controlled trial. BMC Pulmonary Medicine 2011 11:16.

\section{Submit your next manuscript to BioMed Central and take full advantage of:}

- Convenient online submission

- Thorough peer review

- No space constraints or color figure charges

- Immediate publication on acceptance

- Inclusion in PubMed, CAS, Scopus and Google Scholar

- Research which is freely available for redistribution

Submit your manuscript at www.biomedcentral.com/submit
Biomed Central 